\section{Cureus}

Received 01/23/2013

Review began 01/23/2013

Published 02/06/2013

\section{(๑) Copyright 2013}

Isariyawongse et al. This is an open access article distributed under the terms of the Creative Commons Attribution License CC-BY 3.0., which permits unrestricted use, distribution, and reproduction in any medium, provided the original author and source are credited.

\title{
Coil Embolization of a Giant Renal Artery Aneurysm
}

\author{
Justin P. Isariyawongse $^{1}$, Lee E. Ponsky ${ }^{2}$, Joseph P. Lipuma ${ }^{3}$ \\ 1. Center for Urologic Oncology \& Minimally Invasive Therapies, Urology Institute, University Hospitals \\ Case Medical Center, 11100 Euclid Avenue, Cleveland, Ohio 44106, Center for Urologic Oncology \& \\ Minimally Invasive Therapies, Urology Institute, University Hospitals Case Medical Center, 11100 Euclid \\ Avenue, Cleveland, Ohio 44106 2. University Hospitals Case Western Reserve University School of \\ Medicine 3. Department of Radiology, University Hospitals Case Medical Center, Cleveland, Ohio
}

$\square$ Corresponding author: Lee E. Ponsky, lee.ponsky@uhhospitals.org

Disclosures can be found in Additional Information at the end of the article

\section{Abstract}

Renal artery aneurysms (RAAs) are rare but harbor serious consequences if they rupture. When determining if a RAA should be treated, physicians consider aneurysm size as it pertains to the likelihood of rupture. Giant RAAs have rarely been reported in the literature and have usually been managed by open aneurysmectomy and nephrectomy. In the present case, we report a 63year-old male incidentally found to have a giant aneurysm of his right segmental and interlobar arteries on computed tomography (CT). The patient underwent successful uncomplicated transcatheter coil embolization of his aneurysm and maintained no flow into the aneurysm with normal renal function seven years following the procedure.

Categories: Radiology, Urology

Keywords: coil embolization, renal artery aneurysm

\section{Introduction}

Renal artery aneurysms (RAAs) are reported to occur in approximately $0.1 \%$ of the general population [1]. In most cases, RAAs are discovered incidentally and do not require treatment. However, in cases where there is increased risk of rupture - for example, aneurysms discovered in pregnant women and aneurysms greater than $2 \mathrm{~cm}$ in diameter - intervention is indicated [2]. For patients with an extraparenchymal aneurysm, this may entail aneurysmectomy with either patch angioplasty or aortorenal bypass; additionally, surgical management of an intraparenchymal aneurysm may require partial or total nephrectomy [3]. Recently, however, RAAs have been successfully managed by various methods of transcatheter embolization. In this report, we present the case of a 62-year-old male with a giant aneurysm of his right segmental and interlobar artery, who underwent successful endovascular exclusion of the aneurysm by transcatheter coil embolization.

\section{Case Presentation}

A 62-year-old male presented to his primary care physician for routine follow-up of his preexisting sarcoidosis. At that time, computed tomography (CT) of the chest was obtained, incidentally demonstrating a right renal lesion that, on ultrasound, was noted to be hypoechoic and to measure $3.8 \mathrm{~cm} \times 4.1 \mathrm{~cm} \times 3.9 \mathrm{~cm}$ in size. Doppler imaging revealed both an arterial and a venous component that was concerning for either a renal artery aneurysm (RAA) or an arteriovenous malformation. 


\section{Cureus}

The patient was referred to our institution, at which time he reported no history of gross hematuria or localized flank pain. The gentleman's family history was, however, significant for a brother who had died of a cerebral aneurysm around the age of 40 . The patient was normotensive, and his renal function, urine cultures, and cytology were normal. However, a dedicated triple phase CT scan of the kidneys confirmed the presence of a $5.8 \mathrm{~cm} \times 3.8 \mathrm{~cm}$ vascular mass at the right renal hilum with possible contribution from branches of the right renal artery (Figure 1).

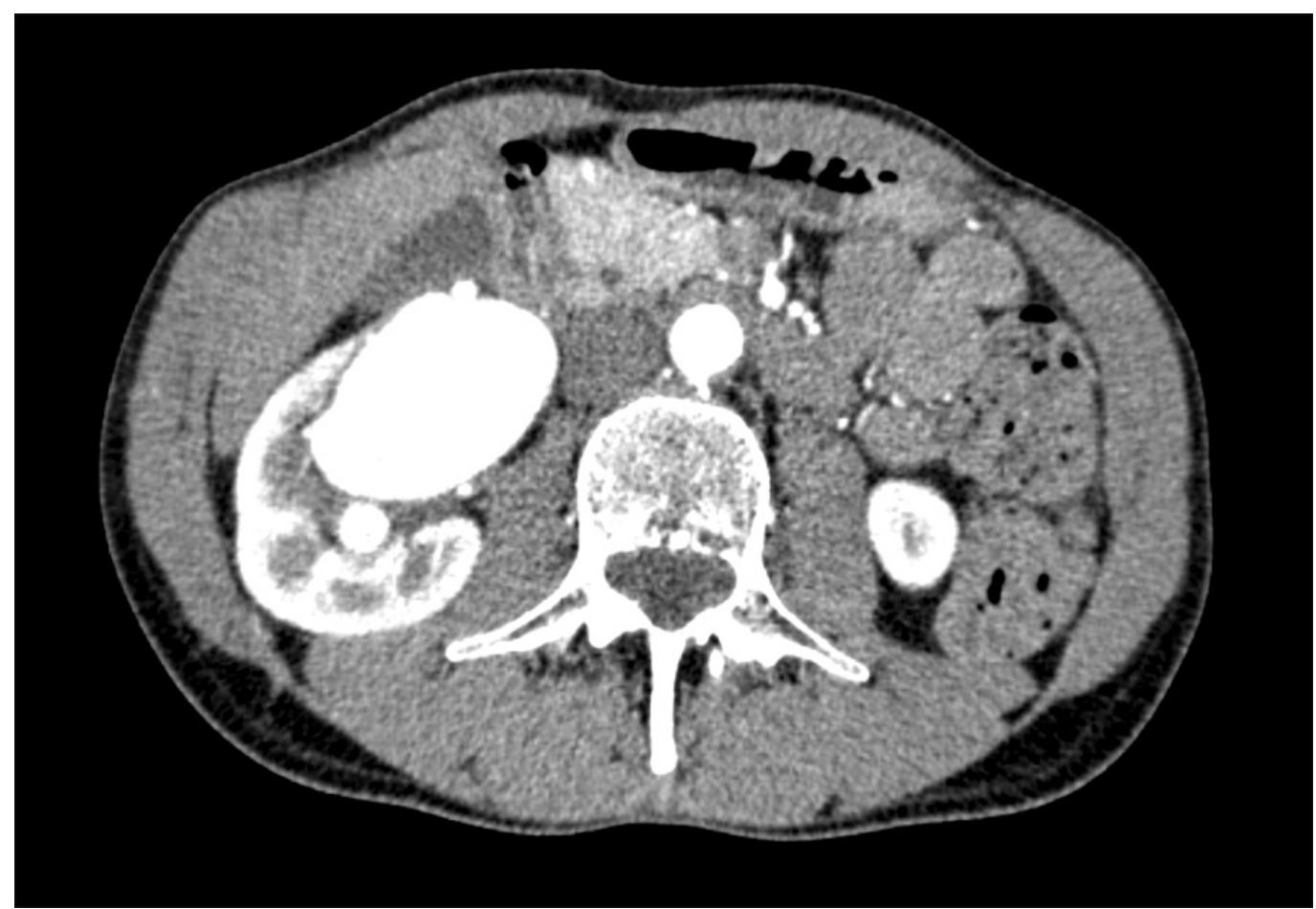

FIGURE 1: Pre treatment CT

A follow-up angiogram detailed a large hyperattenuating aneurysm at the right renal hilum appearing to originate from a lower pole branch of the renal artery In an effort to minimize the loss of normal parenchyma, it was recommended that the patient undergo a coil embolization procedure to facilitate thrombosis of the aneurysm. If he were to fail this management, plans were made to proceed with either surgical repair of the aneurysm or a right nephrectomy.

Approximately three weeks after angiography, the patient returned for coil embolization. Under conscious sedation, a 6-French anesthetic sheath was inserted into the right common femoral artery. Over a guidewire, a 5-French $\mathrm{C} 1$ catheter was inserted through the sheath, facilitating selective arteriography of the right main and divisional renal arteries. Sequential distal catheterizations and subsequent embolizations of the right lower pole segmental and interlobar arteries were performed via a microcatheter passed through the C1 catheter. A single Nestor coil $14 \mathrm{~cm}$ in length and $8 \mathrm{~mm}$ in diameter was deployed under CT-guidance, accomplishing coil embolization of the right lower pole segmental artery and the right lower pole interlobar artery. Successful occlusion of these supply vessels to the aneurysm was confirmed by post-procedural arteriogram and CT (Figure 2). 


\section{Cureus}

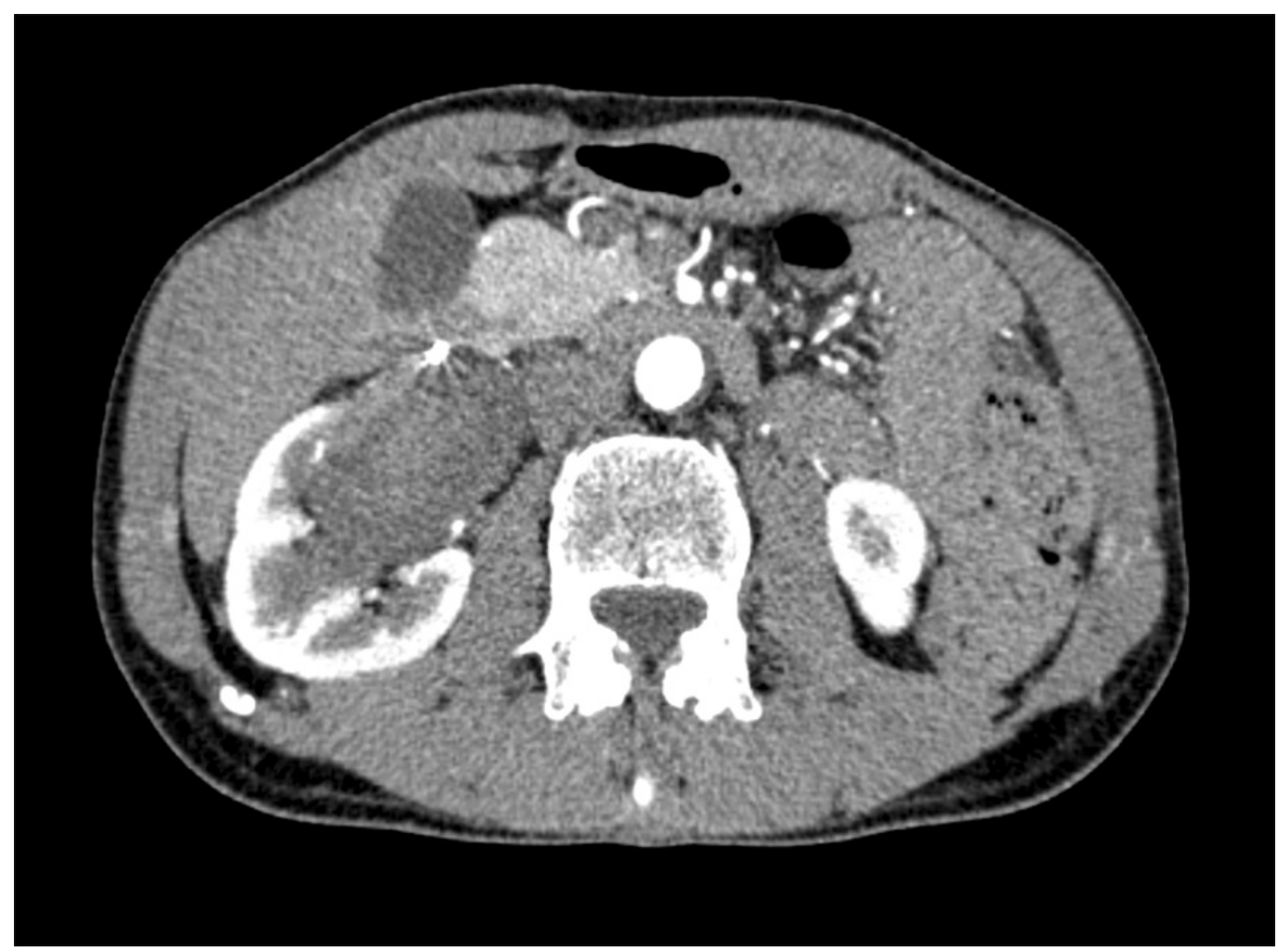

FIGURE 2: CT immediately following embolization

It was estimated that less than $30 \%$ of renal parenchymal loss was suffered at the time of embolization. The patient's immediate post-procedural course was uncomplicated, and he has remained without abdominal or flank pain on follow-up. His renal function panels have remained within the normal range. Two weeks following embolization, CT angiography demonstrated complete absence of flow to the previous aneurysmal site with preservation of nephrogenic and corticomedullary enhancement within the right kidney. Additionally, serial CT angiography performed four months, eight months and yearly (Figure 3) after embolization has demonstrated an absence of flow to the former aneurysmal sac, a decrease in its caliber, and interval atresia of the vessels proximal to the coil location. Currently, seven years from the time of the embolization, the patient maintains absence of flow into the aneurysmal sac (Figure $4)$. 


\section{Cureus}

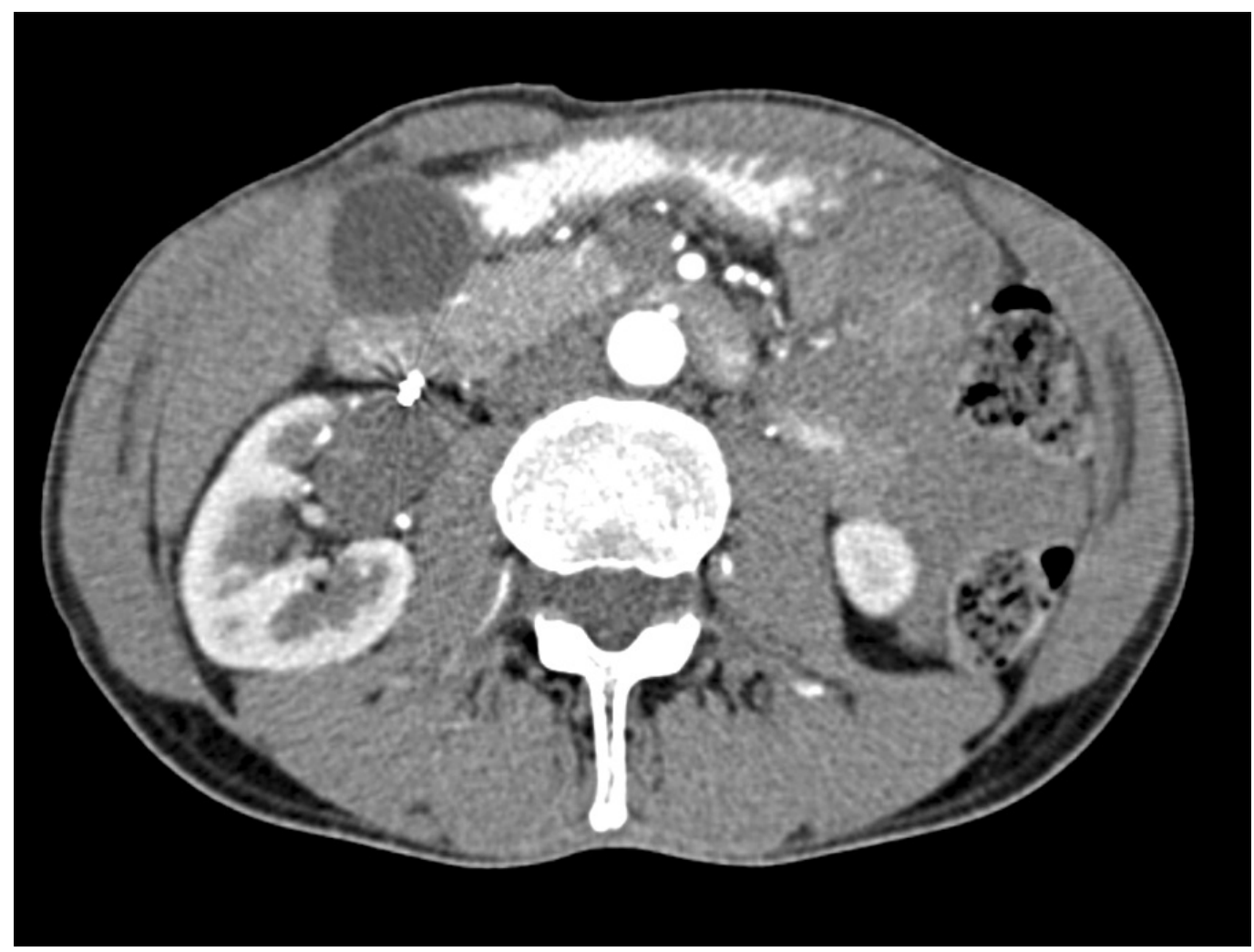

FIGURE 3: Follow up CT at 4 years post embolization

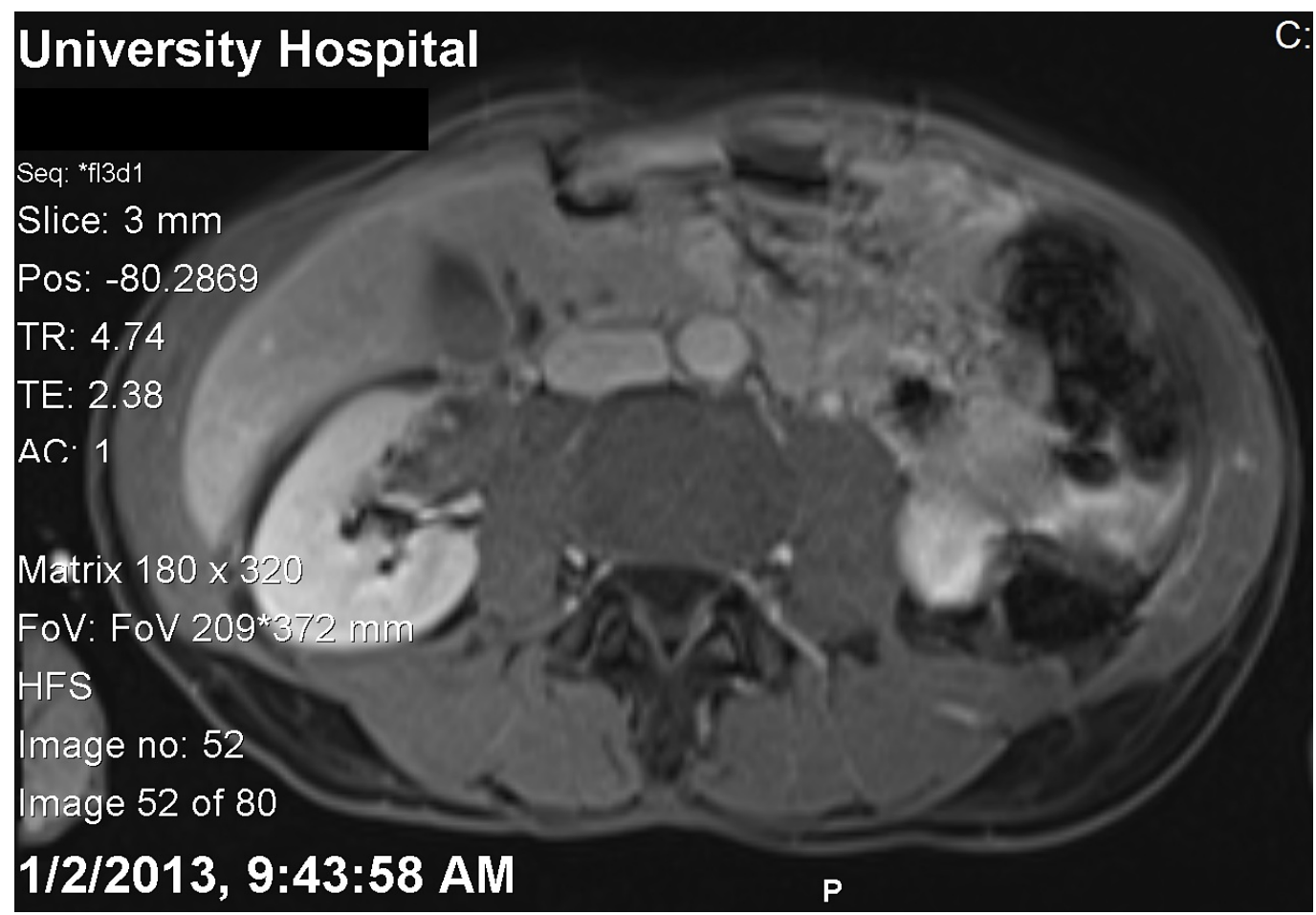

FIGURE 4: Follow up CT at 4 years post embolization 
The patient will be followed at our institution annually with scheduled office visits and CT angiography.

\section{Discussion}

While renal artery aneurysms (RAAs) are discovered in as many as $1.0 \%$ of patients undergoing abdominal aortic angiography [4], their overall incidence is more rare, occurring in just $0.1 \%$ of the general population [1]. While the evaluation for RAAs may be prompted by symptoms such as hematuria, flank pain, or hypertension, the frequency with which asymptomatic RAAs are being discovered as incidental radiologic findings is increasing, owing to more frequent use of magnetic resonance imaging (MRI), computed tomography (CT), and arteriography.

The need to intervene when a RAA is discovered is largely predicated on the likelihood of the aneurysm to rupture. Intervention is indicated in women who are pregnant because changes related to pregnancy (e.g. increased intra-abdominal pressure, increased blood flow, and vascular changes) predispose this population to RAA rupture [5.]; indeed, RAA rupture is a surgical emergency with a reported mortality rate of $80 \%$ although this number is less than $10 \%$ in nonpregnant patients [6-7]. Thus, intervention is indicated for RAAs in the following situations: 1) RAAs greater than $2 \mathrm{~cm}$ in diameter, 2) those which have demonstrated a tendency for enlargement, 3) symptomatic RAAs (e.g. hematuria, flank pain) that may be indicative of an expanding aneurysm, 4) RAAs in women who are pregnant or expect to be pregnant, and 5) those associated with renovascular hypertension [2, 8]. Among these indications, size of the aneurysmal sac is the most frequently debated. Whereas $2.0 \mathrm{~cm}$ is frequently cited as the threshold to determine when to treat a RAA, there have been documented cases of RAAs smaller than this size rupturing [9].

The average size of a RAA in a series of 168 patients with 252 aneurysms was $1.3 \mathrm{~cm}$ in those who were observed, and $1.5 \mathrm{~cm}$ in those who underwent definitive management [7]. Giant aneurysms such as the one demonstrated in our case have only occasionally been reported in the literature. They represent the type of RAAs that are not only above the threshold for recommended repair but are also ones that might more frequently be managed by nephrectomy. To the best of our knowledge, this is the second largest renal artery aneurysm successfully managed with coil embolizationTrocciola et al have previously described a $13 \mathrm{~cm}$ RAA with a concurrent arteriovenous fistula that underwent coil embolization [10].

The approach to RAA repair is often based upon the location of the aneurysm. Aneurysmectomy combined with either patch angioplasty or aortorenal bypass has frequently been undertaken for those RAAs in extraparenchymal locations. Additionally, surgical management of an intraparenchymal aneurysm may require partial or total nephrectomy [3]. In regard to size, one study reports that there was no significant difference between the size of aneurysms that were observed and those that underwent repair [7]. However, the few reported cases of giant RAAs in the literature have frequently required open repair and nephrectomy [11-14].

\section{Conclusions}

In the case presented in this report, a giant RAA was successfully managed by transcatheter embolization and deployment of a single Nestor coil. Coil embolization in this case was a technically feasible procedure that spared the patient an open revascularization procedure or nephrectomy. Seven years following the procedure, continued exclusion of the aneurysm has been demonstrated, and the patient has had no complications nor demonstrable compromise of his renal function. We conclude that coil embolization for the management of RAAs is a straightforward alternative to surgery that can be employed safely and with good long-term results and that the size of the aneurysm is not a prohibitive factor in selecting this method of treatment. 


\section{Additional Information \\ Disclosures}

Human subjects: Consent was obtained by all participants in this study. Conflicts of interest: In compliance with the ICMJE uniform disclosure form, all authors declare the following:

Payment/services info: All authors have declared that no financial support was received from any organization for the submitted work. Financial relationships: All authors have declared that they have no financial relationships at present or within the previous three years with any organizations that might have an interest in the submitted work. Other relationships: All authors have declared that there are no other relationships or activities that could appear to have influenced the submitted work.

\section{References}

1. Deterling RA: Aneurysms of the visceral arteries. J Cardiovasc Surg. 1981, 12:309-322.

2. Ortenberg J, Novic AC, Straffon RA, et al: Surgical treatment of renal artery aneurysm. Br J Urol. 1983, 55:341-346. 10.1111/j.1464-410X.1983.tb03317.x

3. Stanley JC: Renal artery aneurysm. Current Therapy in Vascular Surgery. Ernst JC \& Stanley JC (ed): Mosby Elsevier Health Science, Ann Arbor; 1995. 813-817.

4. Tham G, Ekelund L, Herrlin K, et al: Renal artery aneurysm: natural history and prognosis . Ann Surg. 1983, 197:348-352. 10.1097/00000658-198303000-00016

5. Soliman KB, Shawky Y, Abbas MM, et al: Ruptured renal artery aneurysm during pregnancy, a clinical dilemma. BMC Urol. 2006, 6:22.

6. Ippolite JA, LeVeen HH: Treatment of renal artery anurysm. J Urol. 1960, 83:10-16.

7. Henke PK, Cardneau JD, Welling TH, et al: Renal artery aneurysms: a 35-year clinical experience with 252 aneurysms in 168 patients. Ann Surg. 2001, 234:454-463. 10.1097/00000658-200110000-00005

8. Pershad A, Heuser R: Renal artery aneurysm: successful exclusion with a stent graft . Catheter Cardiovasc Interv. 2004, 61:314-316. 10.1002/ccd.10789

9. Reiher L, Grabitz K, Sandmann W: Reconstruction for renal artery aneurysm and its effect on hypertension. Eur J Vasc Endovasc Surg. 2000, 20:454-456. 10.1053/ejvs.2000.1216

10. Trocciola SM, Chaer RA, Lin SC, et al: Embolization of renal artery aneurysm and arteriovenous fistula: a case report. Vasc Endovascular Surg. 2005, 39:525-529. $10.1177 / 153857440503900610$

11. Kato T, Takagi H, Ogaki K, et al: Giant renal artery aneurysm with arteriovenous fistula . Heart Vessels. 2006, 21:270-272.

12. Chiesa R, Melissano G, Castellano R: Giant renal artery aneurysm. J Vasc Surg. 2004, 40:1245. 10.1016/j.jvs.2004.01.026

13. Puri D, Sharma R, Samra N, et al: Surgical management of large renal artery aneurysm - case report. Ind J Thoracic Cardiovasc Surg. 2004, 20:210-212. 10.1007/s12055-004-0091-y

14. Sajja LR, Sitaram Reddy B, Sahariah S, et al: Giant aneurysm of renal artery: surgical management. Asian Cardiovasc Thorac Ann. 2002, 10:176-177. 10.1177/021849230201000222 